\title{
The Emerging Role of Telehealth in Antimicrobial Stewardship: A Systematic Review and Perspective
}

\author{
Jacob Pierce ${ }^{1^{*}}$ \\ Michael P. Stevens ${ }^{1}$
}

\author{
Address \\ ${ }^{1}$ Department of Internal Medicine, Division of Infectious Diseases, Virginia \\ Commonwealth University School of Medicine, 1000 E Marshall St., Richmond, \\ VA 23298, USA \\ Email: Jacob.Pierce@vcuhealth.org
}

Published online: 28 December 2021

๑ The Author(s), under exclusive licence to Springer Science+Business Media, LLC, part of Springer Nature 2021

Keywords Antimicrobial stewardship - Telehealth - Telestewardship - Antimicrobial resistance

\section{Abstract}

Purpose of Review The purpose of this article is to critically evaluate the available literature on telehealth and antimicrobial stewardship.

Recent Findings There is limited evidence regarding the role of telehealth within the discipline of antimicrobial stewardship. A review of the available literature suggests remote antimicrobial stewardship programs conducted via telehealth can decrease antimicrobial consumption. A direct comparison between traditional antimicrobial stewardship programs and remote antimicrobial stewardship programs is lacking.

Summary Telehealth is a promising tool for expanding antimicrobial stewardship, especially in small rural or community hospitals. Trust, team, and technology, the three Ts of remote antimicrobial stewardship derived from themes within the available literature, can serve as a framework for developing a remote antimicrobial stewardship program. 


\section{Introduction}

Telehealth is an emerging field that has a potential role in expanding antimicrobial stewardship to small and critical access hospitals. This review seeks to summarize the available literature on the utilization of telehealth for providing antimicrobial stewardship. The 2019 United States Centers for Disease Control and Prevention (CDC) core elements for hospital antibiotic stewardship suggest the use of off-site or remote Infectious Diseases (ID)-trained providers when local resources are not available $[1 \bullet \bullet$. A recent report published in the Annals of Internal Medicine found that in 2017 roughly $80 \%$ of counties in the USA did not have an ID physician [2]. A 2012 survey showed that $40 \%$ of Veteran's Affairs Medical Centers with inpatient care did not have full-time ID physicians on staff [3]. Also in 2019, the Centers for Medicare and Medicaid Services (CMS) set a requirement for all acute-care hospitals that utilize Medicare or Medicaid to include antimicrobial stewardship by March 30, 2020 [4••]. The COVID-19 pandemic has highlighted the disparity in access to ID physicians, and novel strategies are needed to meet the demand for ID expertise. Telehealth is a potential solution to increasing ID physician access.

Additionally, the pandemic has brought telemedicine to the forefront for medical providers around the world. As of quarter three of 2020, venture capital funding in telehealth had reached $\$ 9.4$ billion USDalready surpassing the previous annual record of $\$ 8.2$ billion in 2018 [5].

The Infectious Diseases Society of America (IDSA) published a position statement in 2019 on the role of telehealth in the field of Infectious Diseases [6•].
They note the Health Resources Services Administration defines telehealth as "the use of electronic information and telecommunications technologies to support long-distance clinical healthcare, patient and professional health-related education, public health and health administration." According to definitions put forth by the IDSA, telehealth with antimicrobial stewardship would primarily involve asynchronous telemedicine without direct patient communication. The IDSA supports the use of telehealth in antimicrobial stewardship to expand access to antimicrobial stewardship to community hospitals [6•].

A recent systematic review examined the effectiveness of telehealth with ID consultation demonstrating comparable efficacy to in-person consultation based on limited available evidence [7]. A separate review focused more broadly on the application of telehealth in ID practice. They demonstrated similar outcomes in the outpatient setting for patients with HCV, HIV, and tuberculosis, whether clinic visits were conducted in-person or through telehealth services [8]. In 2018 Pottinger et al. provided an overview of the literature and potential value of antimicrobial stewardship conducted via telehealth. They highlighted the need for telehealth in antimicrobial stewardship given the vast disparity between available ID specialists and the number of hospitals. They purported that the fundamentals of a successful stewardship program include building trust with a remote institution through periodic faceto-face communication [9]. To our knowledge, there has been no comprehensive systematic review on the role of telehealth in antimicrobial stewardship.

\section{Methods}

A systematic literature review was performed using PubMed on September 14, 2020. Search terms utilized were "Antimicrobial Stewardship" and "Telehealth" along with "Antibiotic Stewardship" and "Telehealth" and finally "Telestewardship." Only English language articles were included if no translation was available. There was no date range limitation, but all articles were published between 2012 and 2020. Articles were included if they described an antimicrobial stewardship intervention performed by an off-site team utilizing telehealth capabilities such as phone calls, video conferencing, email, 


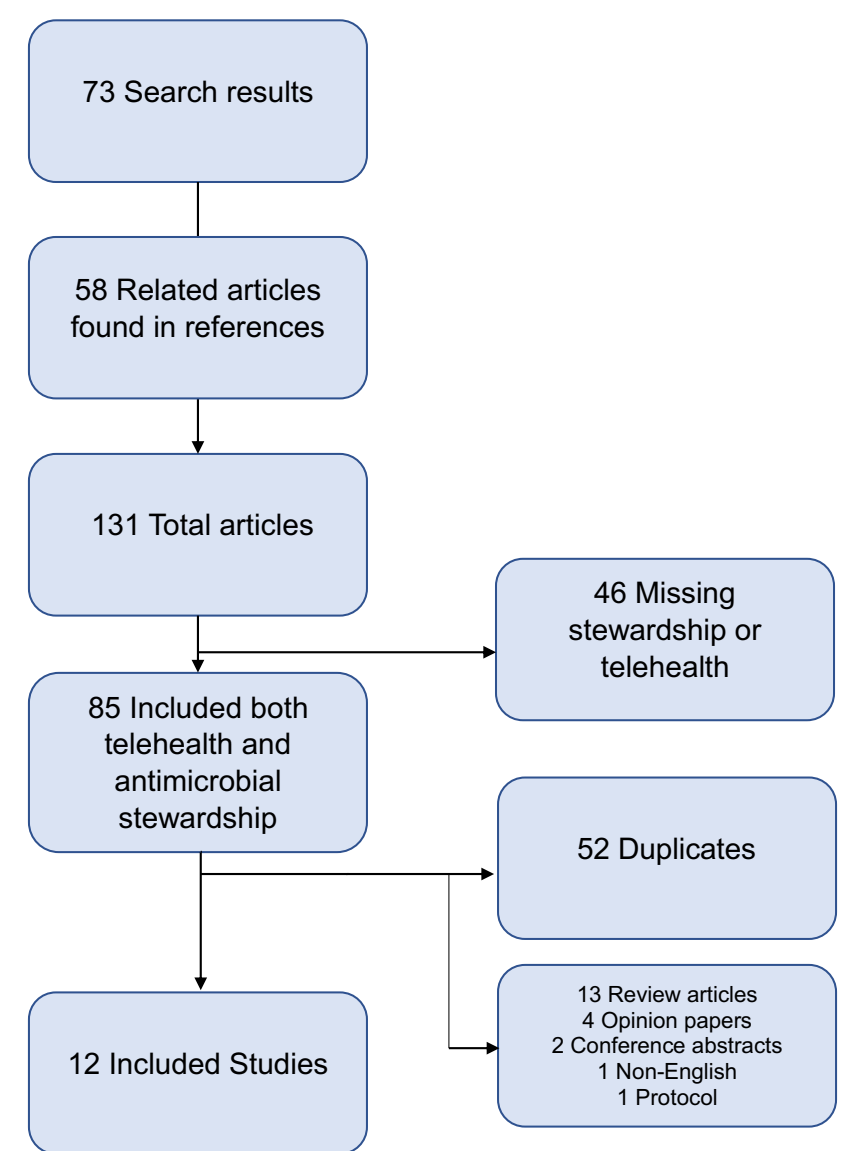

Fig. 1 Literature search results. Seventy-three initial search results were found and an additional 58 related articles were located within the references of these initial references. Studies with horizontal arrows indicate excluded studies. Twelve studies were included in the final analysis

electronic medical record documentation, or other methods of remote communication. All references of included articles were reviewed for the relevant search terms. Conference abstracts were excluded if outcome data could not be determined. See Fig. 1 for the search process. Primary outcomes were included as specified in the article or if not specified (or if multiple primary outcomes were listed) the first listed measurable outcome was labeled as the primary outcome ${ }^{1}$ and all other outcomes were labeled as secondary. Study quality was determined utilizing previously published methodology [10].

Outcomes were sorted based on antimicrobial stewardship intervention. Examples included prospective audit and feedback (PAF), antimicrobial restriction, IV to PO conversion, etc.

\footnotetext{
${ }^{1}$ In one study, "main topic discussed" was inferred to mean primary outcome [18].
} 
A total of 131 articles were reviewed, and 12 met inclusion criteria (Table 1). The quality of included studies was generally low consisting, primarily of observational and quasi-experimental studies with only one randomized controlled trial.

Eight studies were conducted in the USA, two in Brazil, and two in Europe. Primary study sites were community hospitals, three included longterm acute care hospitals, and one study was in the outpatient primary care setting. One study examined a pediatric inpatient population [18], the remainder focused on adults.

The most common ASP intervention was prospective audit and feedback (PAF) included in 58\% (7/12) studies.

The most common primary outcome was antimicrobial consumption reported in 5 of the included studies $[11,13,14,16,20]$. Four of the five studies demonstrated a statistically significant decrease in antimicrobial consumption with the implementation of telehealth antimicrobial stewardship [11, 13, 14, 16].

One study demonstrated a statistically significant decrease in antimicrobial prescriptions for upper respiratory tract infections in the outpatient setting [17]. A separate study examined effects on compliance with local guidelines with a statistically significant increase in the rate of adherence [15]. One study demonstrated a significant decrease in isolation of multidrug resistant organisms [18]. One study found a statistically significant decrease in hospital-acquired Clostridioides difficile infection as a secondary outcome [14].

A single study expressed concern the local private practice ID physician would experience decreased financial compensation from lower consult volume with the implementation of telestewardship and evaluated the effect on ID consultations. They found a $40 \%$ increase in consultation following telehealth antimicrobial stewardship implementation [13]. Another study examined the time commitment required to perform telestewardship and found an average of $3.6 \mathrm{~h}$ per week of time from a remote ID pharmacy resident [22].

Of the examined studies, $83 \%$ (10/12) of telehealth antimicrobial stewardship programs consisted of a remote ID physician (one study utilized an internal medicine trained associated medical director [19], and another consisted of pharmacists [22]) and 25\% (3/12) included a remote pharmacist $[14,20,22]$. One-third (4/12) of local sites created a local ASP team consisting of local providers, infection prevention nurses, and pharmacists $[12,16,17,23]$. The other two-thirds only utilized local providers communicating with the remote ASP team. 


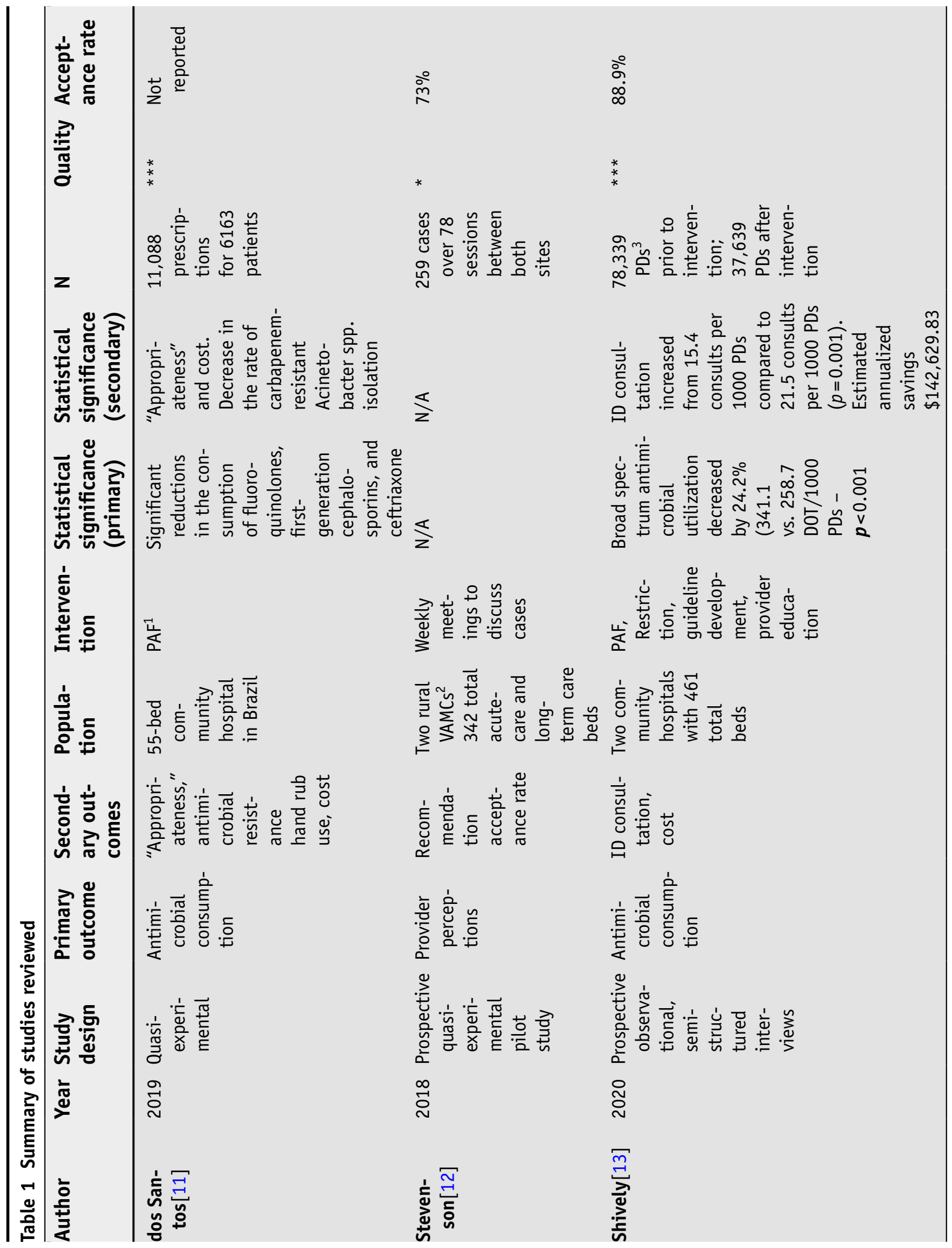




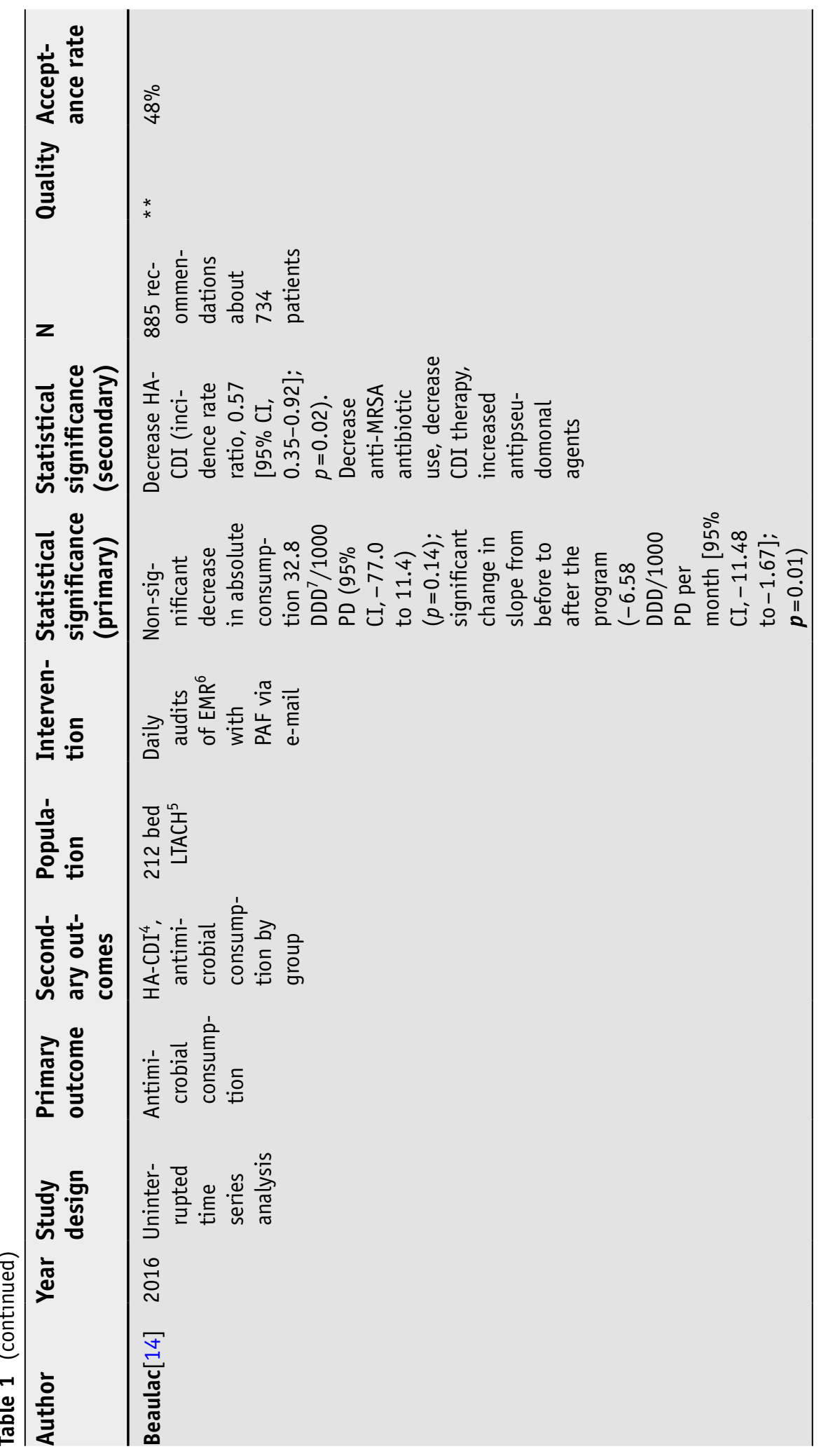




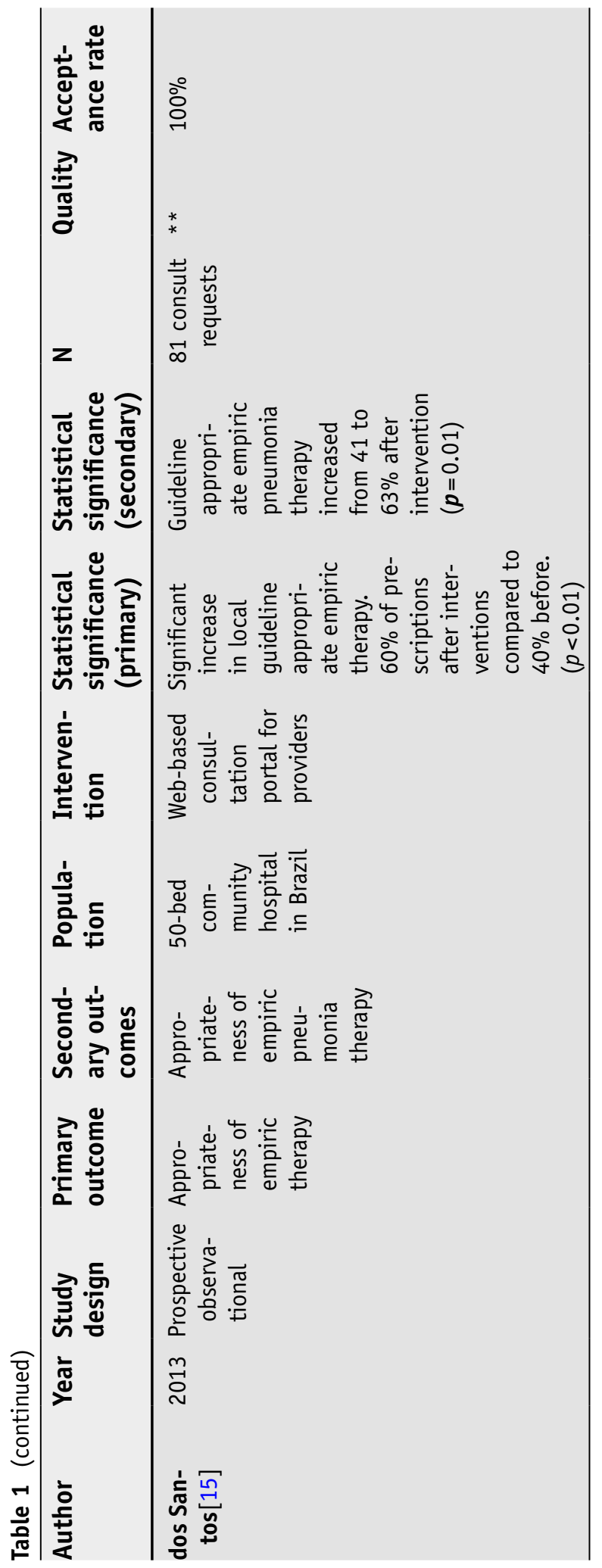




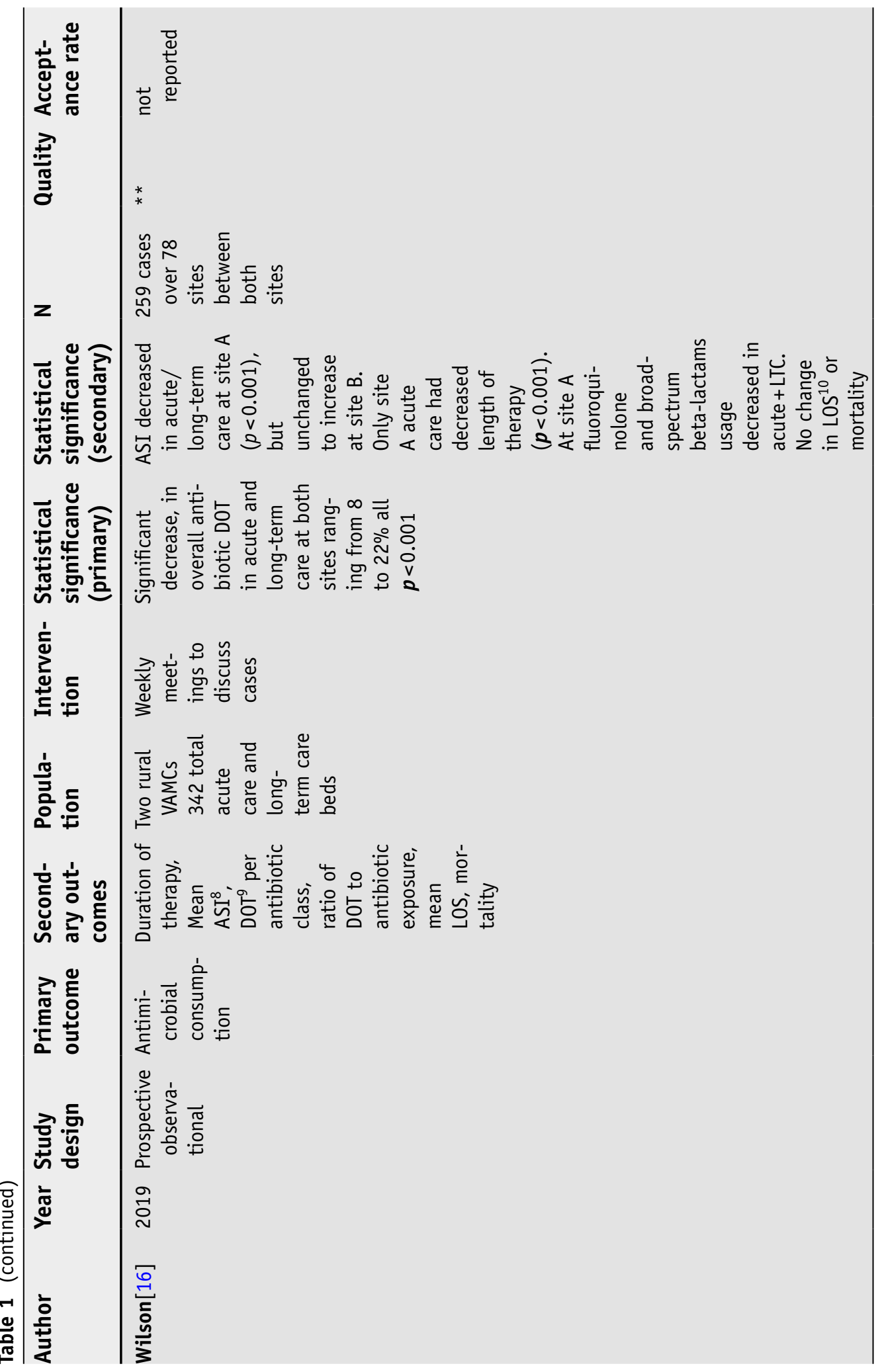




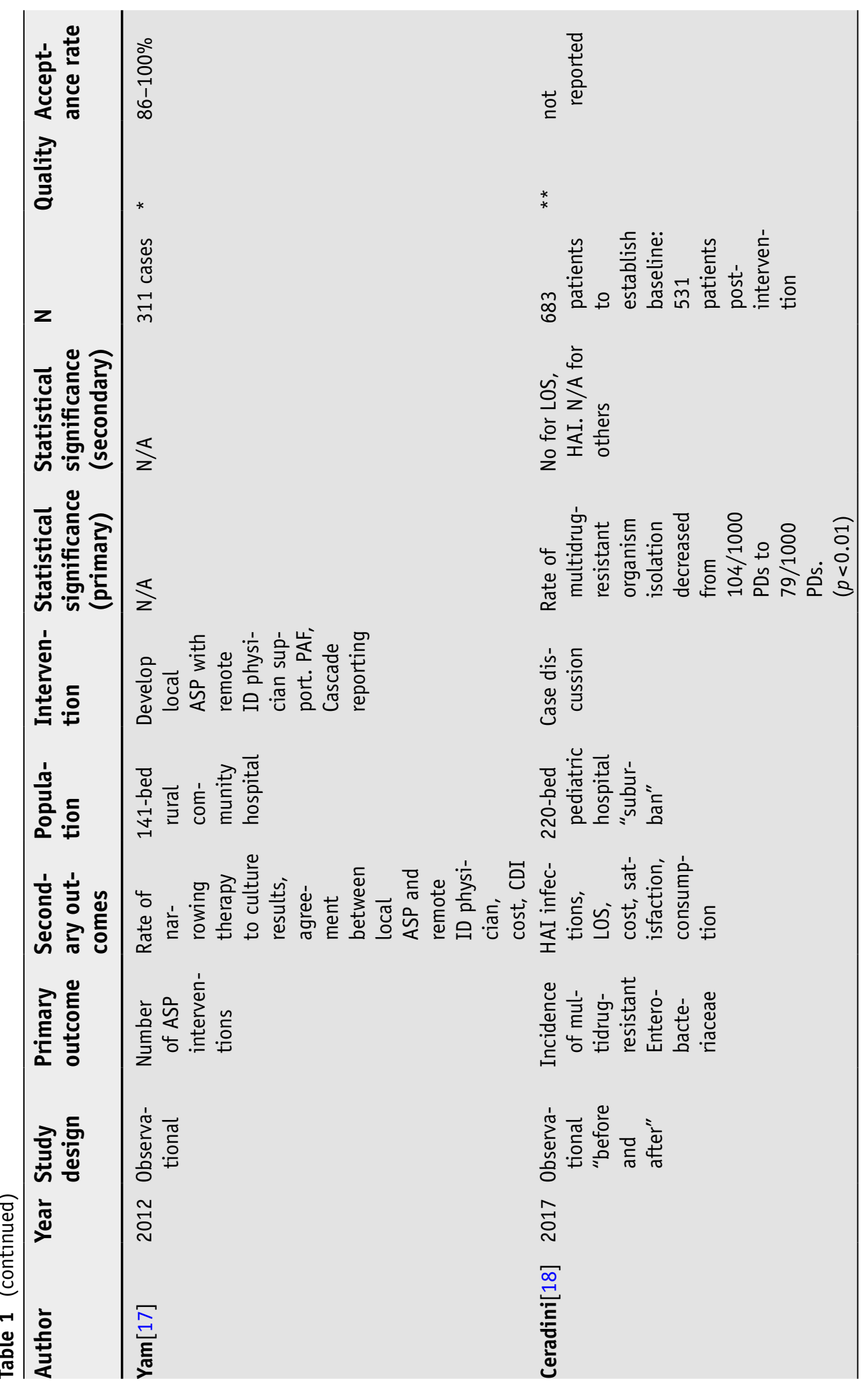




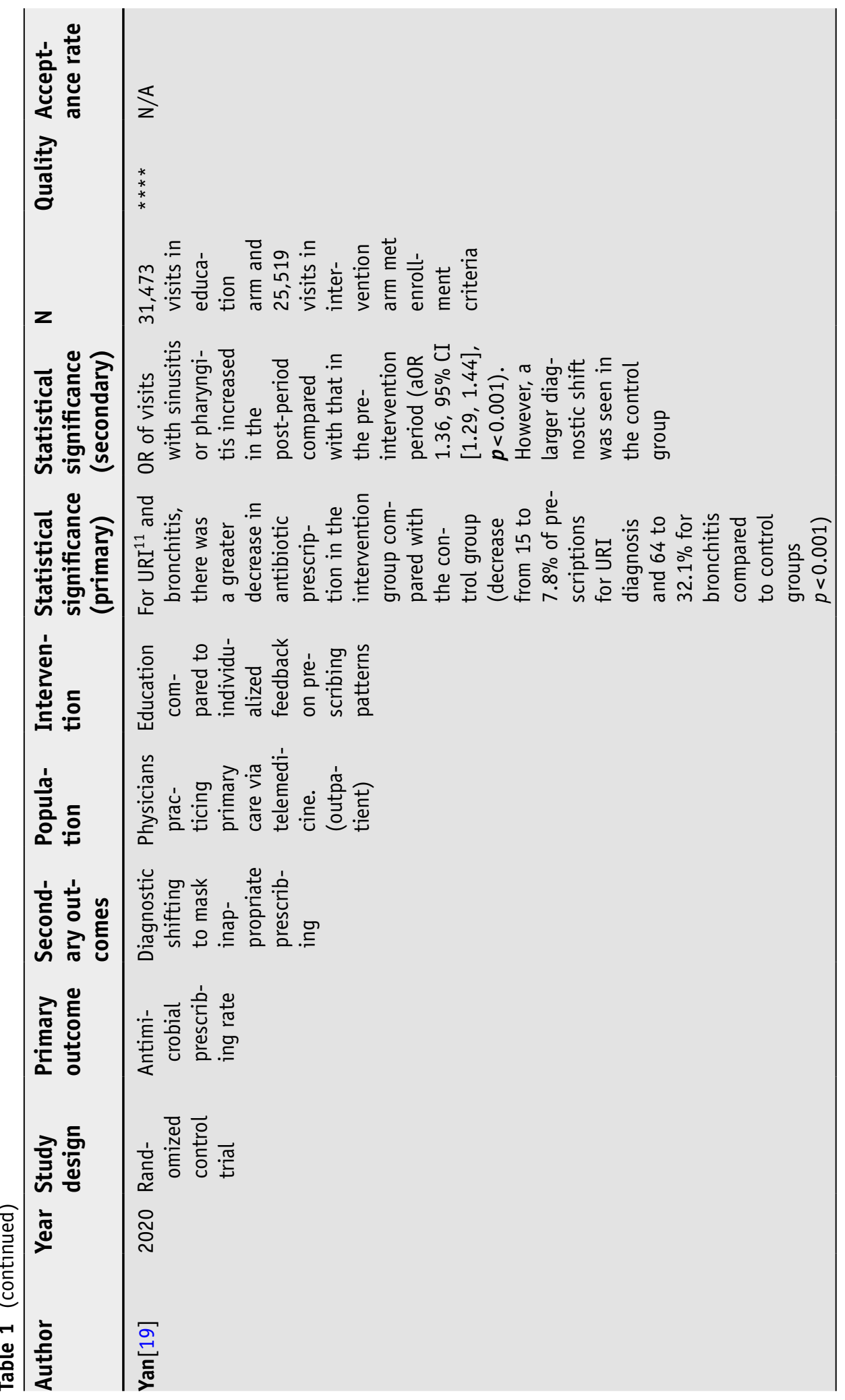




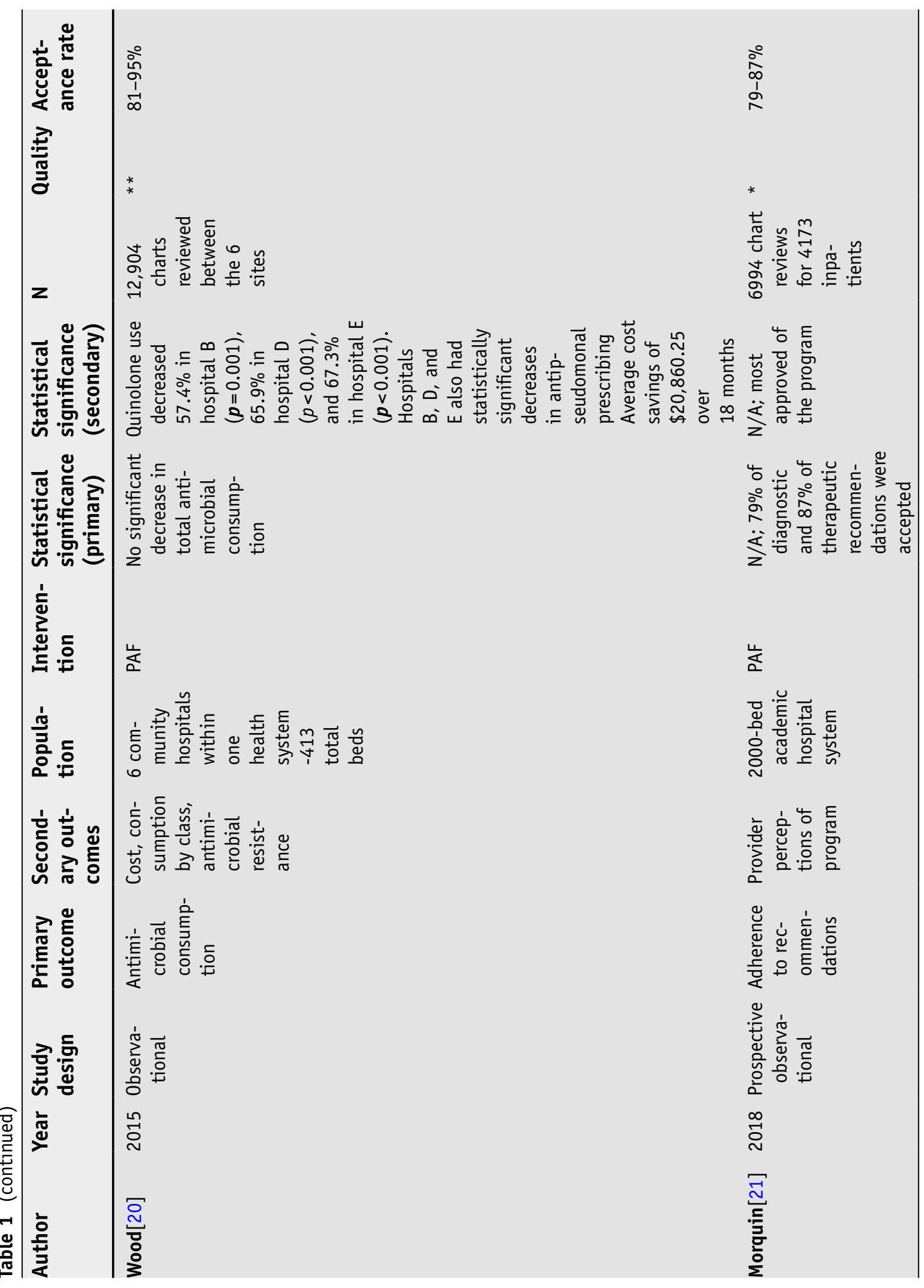




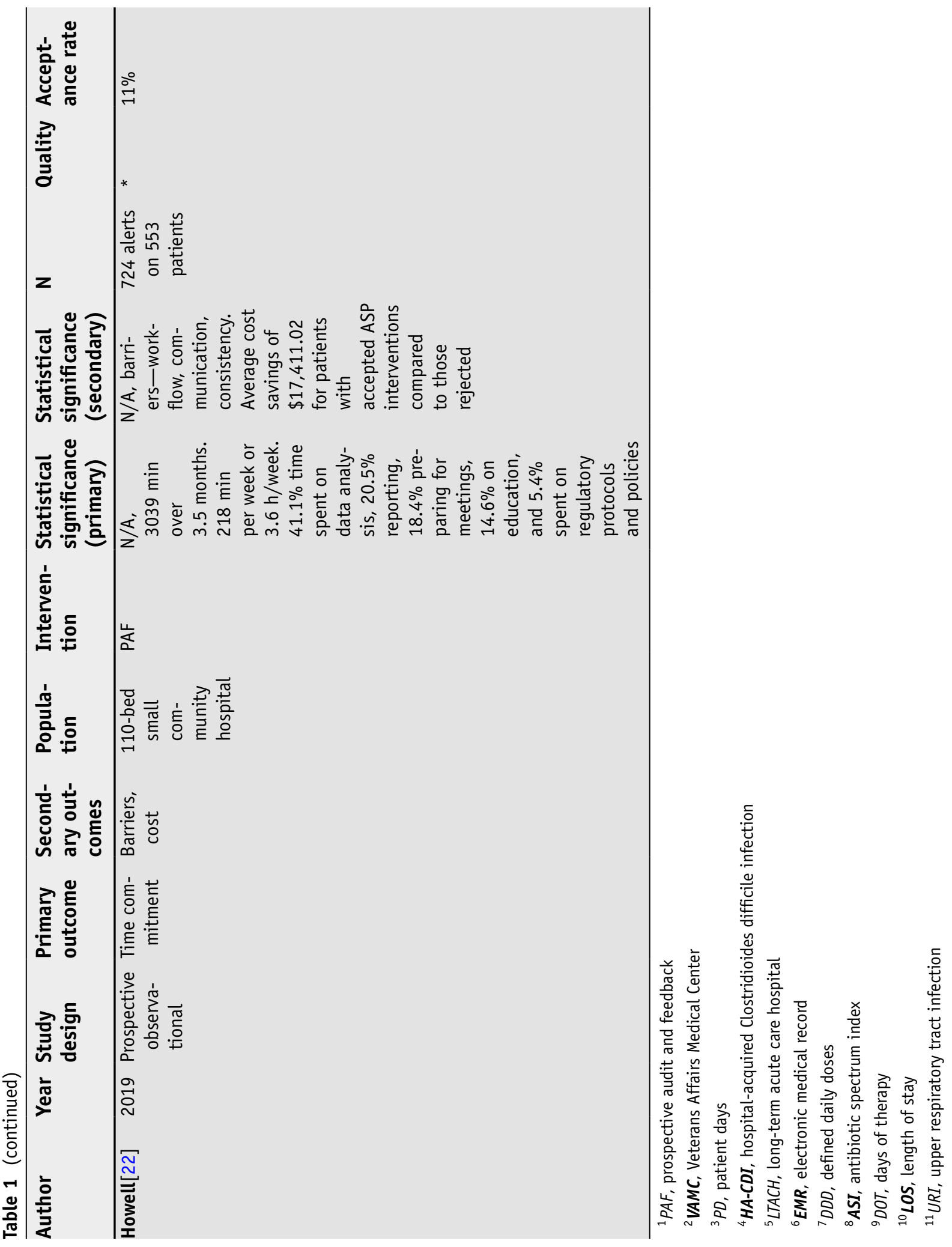


Studies included between 1 and 4 remote ID physicians, 2 studies employed ID-trained pharmacists [14, 22], with one study included pharmacists with unspecified levels of training [20].

The size of hospitals examined ranged from 50 beds up to 2000. The average number of remote ASP providers to hospital beds was one provider for every 234 beds. The number of full-time-equivalents dedicated to remote antimicrobial stewardship was not routinely reported. The exact ratio of remote ASP providers to hospital bed size is unknown although it should be noted that only three of the eleven $(27 \%)$ inpatient studies met the goal for traditional ASP programs of 1.0-1.5 FTEs per 100 hospital beds that has been described $[24,25]$. CMS proposes a minimum of 0.10 physician FTEs along with 0.25 pharmacy FTEs for a "moderate size hospital" (defined as 124 beds), although acknowledges that 1.0 physician FTEs and 0.5 pharmacies FTEs may be more effective $[4 \bullet \bullet]$.

On average, 64.6 interventions were performed per remote provider per month for the 9 studies where this could be determined. It should be noted that the reporting of interventions was not standardized among publications.

\section{Equipment}

The primary modalities of communication were telephone or videoconferencing along with electronic medical record documentation. Two studies from the same author developed web-based platforms [11, 15], three communicated via secure email $[11,14,15]$, and one via secure SMS messaging [15].

There was a wide variance in the provider acceptance rate of remote ASP recommendations ranging from 11 to $100 \%$. The studies with the lowest reported compliance rates lacked face-to-face communication with the local facility-one study relied on e-mail only communication providing a $48 \%$ acceptance rate [14]. An 11\% acceptance rate was seen when the remote ASP personnel communicated via TheraDoc with a local pharmacist and had no direct communication with frontline providers [22]. The remaining studies that reported on acceptance rates included some form of face-to-face communication with local providers and had acceptance rates ranging from 73 to $100 \%$. Among the three studies that developed a local antimicrobial stewardship team and provided acceptance rates, the average acceptance rate was $86.6 \%$. Among the 5 studies that did not create a local ASP, the average was $66.5 \%$. It is notable that the studies not employing local ASP representatives had significantly more variance of acceptance rates and included the two lowest acceptance rates of 11 and $48 \%$. 


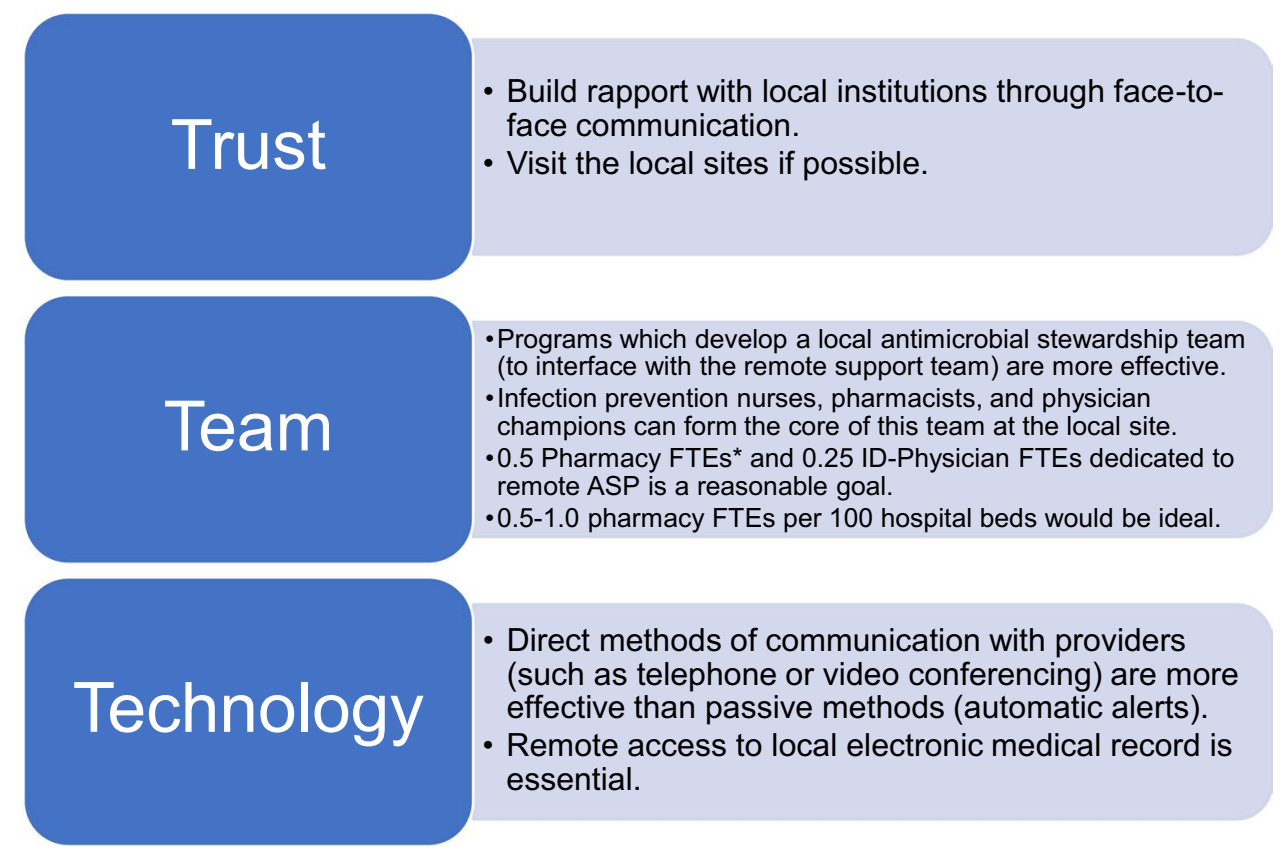

Fig. 2 The three Ts of remote antimicrobial stewardship: trust, team, and technology. *FTE, fulltime equivalent

\section{Financial Impact}

A potential concern regarding telehealth intervention would be decreased financial compensation related to a decrease in local ID consultation. The one study that examined this demonstrated an increase in local consultation. No study reported a financial loss. Three studies reported on annual antimicrobial purchasing cost savings ranging from $\$ 13,907$ to $\$ 142,630$. One study reported a $\$ 17,411$ savings in total hospital charges including, $\$ 10,073$ in pharmacy charges over 4 months. No study directly addressed the cost of establishing or maintaining a telehealth ASP.

\section{Discussion}

Three primary themes which emerged from the review of the available literature are described in Fig. 2. Establishing relationships and building trust with the local institution are frequently cited as vital to success in the examined articles. Examples included visiting with the local facility on a periodic basis, hosting grand rounds on topics regarding antimicrobial stewardship, or monthly teleconference meetings with local stewardship champions.

Another key element in the formation of remote antimicrobial stewardship is the components of the team. Infectious diseases expertise is critical 
to successful antimicrobial stewardship. Recommending infectious diseases consultation is a common ASP intervention. The one study article that mentioned the presence of a local ID provider prior to the implementation noted an increase in consultation [13], while in the remaining studies the remote ASP served as the primary medium of ID expertise.

According to data from the National Residency Matching Program for 2021, there were 365 applicants for 416 open positions in infectious diseases. Although this was an increase from the year prior, a quarter of programs reported an unfilled position [26]. This suggests an increase in the ID workforce is not on the immediate horizon and novel strategies are needed to meet ID expertise for stewardship staffing requirements. Telestewardship is well positioned to meet these needs by extending available ID expertise. When on-site ID consultation is not available, access to remote ID-trained physicians in conjunction with local antimicrobial stewardship champions would greatly facilitate the work of a telestewardship program.

Although additional data are required to establish with certainly, there is a signal suggesting the formation of a local ASP team is associated with a greater acceptance rate of remote ASP team recommendations. According to the National Antimicrobial Stewardship Task Force, a study of the time required to complete various ASP activities within 12 VAMCs suggested a minimum or 0.25 ID physician full-time equivalents (FTEs) and 0.5 pharmacy FTEs dedicated to an ASP regardless of hospital size with a goal of 1.0 ID-trained pharmacy FTEs per 100 patient beds for a robust ASP. It should be noted that only $21 \%$ of their facilities had more than one pharmacy FTE dedicated to ASP despite 8 of the facilities having 200 or more beds [24]. A separate study examining a survey of 244 US institutions found that institutions employing less than 0.5 dedicated FTEs to antimicrobial stewardship were less likely to be able to perform the most effective ASP activities such as prospective audit and feedback. They also demonstrated a 1.5 -fold increase in achieving primary outcomes measures such as cost savings, decreased antimicrobial consumption, or decreased multi-drug resistant organisms per each 0.5 pharmacies FTEs dedicated to ASP. They proposed a minimum of 1.0 pharmacy FTEs with 0.5 physician FTEs with roughly 1 additional pharmacy FTE per 500 beds [25]. Although there is no specific guidance for telestewardship, these FTE targets are reasonable goals for a remote ASP.

Certain technological capabilities are required to conduct antimicrobial stewardship via telehealth. According to publicly released statements from the CMS, telehealth encounters have increased by $2600 \%$ in 2020 compared to 2019 [27]. This suggests a potential increase in telehealth capabilities developed during the COVID-19 pandemic that could be utilized for telestewardship activities post-pandemic.

Several limitations were noted in the included studies. For the study with the lowest acceptance rate [22], it is conceivable that this may have been related to the reluctance of the local clinical pharmacist to accept recommendations from a pharmacy resident. Robust telestewardship models ideally would include services provided by ID-trained pharmacists and physicians; any learners involved ideally would be precepted by staff clinicians. Although several studies commented on cost savings, the direct cost of establishing the remote antimicrobial stewardship programs was not discussed. 
The lack of direct comparison between remote antimicrobial stewardship and traditional antimicrobial stewardship programs is notable and is a key target for future research.

\section{Conclusion}

Telehealth is a rapidly expanding field and antimicrobial stewardship programs are well-positioned to take advantage of this growing technology. Data in the existing literature are not robust but suggest facilitators include local antimicrobial stewardship champions as well as access to infectious diseases consultants (either locally or via telehealth services). More research is needed to fully characterize the role of telehealth in antimicrobial stewardship, but existing data suggest this may be a promising mechanism to support hospitals that cannot create robust local programs.

\section{Declarations}

\section{Conflict of Interest}

The authors declare no competing interests.

\section{References}

Papers of particular interest, published recently, have been highlighted as:

- Of importance

•- Of major importance

1.• CDC. Implementation of antibiotic stewardship core elements at small and critical access hospitals [Internet]. Cent. Dis. Control Prev. Natl. Cent. Emerg. Zoonotic Infect. Dis. 2020 [cited 2020 Nov 9]. Available from: https://www.cdc.gov/antibioticuse/core-elements/small-critical.html.

Guidance on critical components of an antimicrobial stewardship program.

2. Walensky RP, McQuillen DP, Shahbazi S, Goodson JD. Where is the ID in COVID-19? Ann Intern Med [Internet]. 2020;173:587-9. Available from: https:// www.acpjournals.org/doi/https://doi.org/10.7326/ M20-2684

3. Chou AF, Graber CJ, Jones M, Zhang Y, Goetz MB, Madaras-Kelly K, et al. Characteristics of antimicrobial stewardship programs at veterans affairs hospitals: results of a nationwide survey. infect control hosp epidemiol. 2016;2016:1-8.
4.•• CMS. Medicare and medicaid programs; regulatory provisions to promote program efficiency, transparency, and burden reduction; fire safety requirements for certain dialysis facilities; hospital and critical access hospital (CAH) changes to promote innovation, flexibility, and improvement in patient care [Internet]. 2019. Available from: https://www.feder alregister.gov/documents/2019/09/30/2019-20736/ medicare-and-medicaid-programs-regulatory-provi sions-to-promote-program-efficiency-transparen cy-and.

Regulatory guidance for ensuring compliance when establishing an antimicrobial stewardship program.

5. Rock Health. Q3 2020: a new annual record for digital health (already) [Internet]. 2020 [cited 2020 Nov 11]. Available from: https://rockhealth. com/reports/q3-2020-digital-health-funding-alrea dy-sets-a-new-annual-record/ 
6. Young JD, Abdel-Massih R, Herchline T, McCurdy L, Moyer KJ, Scott JD, et al. Infectious diseases society of America position statement on telehealth and telemedicine as applied to the practice of infectious diseases. Clin Infect Dis. 2019;68:1437-43.

IDSA perspective on role for telehealth within infectious disease including antimicrobial stewardship.

7. Burnham JP, Fritz SA, Yaeger LH, Colditz GA. Telemedicine infectious diseases consultations and clinical outcomes: a systematic review. Open Forum Infect Dis. 2019;6:1-6.

8. Parmar P, Mackie D, Varghese S, Cooper C. Use of telemedicine technologies in the management of infectious diseases: a review. Clin Infect Dis. 2015;60:1084-94.

9. Pottinger PS, Kassamali Z, Wright TC, Scott JD, Martinez-Paz N, Lynch JB. Tele-antimicrobial stewardship in action. Curr Treat Options Infect Dis. Current Treatment Options in Infectious Diseases; 2018;10:229-39.

10. Rittmann B, Stevens MP. Clinical decision support systems and their role in antibiotic stewardship: a systematic review. Curr Infect Dis Rep. Current Infectious Disease Reports; 2019;21.

11. dos Santos RP, Dalmora CH, Lukasewicz SA, Carvalho $\mathrm{O}$, Deutschendorf $\mathrm{C}$, Lima R, et al. Antimicrobial stewardship through telemedicine and its impact on multi-drug resistance. J Telemed Telecare. 2019;25:294-300.

12. Stevenson LD, Banks RE, Stryczek KC, Crnich CJ, Ide EM, Wilson BM, et al. A pilot study using telehealth to implement antimicrobial stewardship at two rural veterans affairs medical centers. Infect Control Hosp Epidemiol. 2018;39:1163-9.

13. Shively NR, Moffa MA, Paul KT, Wodusky EJ, Schipani BA, Cuccaro SL, et al. Impact of a telehealth-based antimicrobial stewardship program in a community hospital health system. Clin Infect Dis. 2020;71:539-45.

14. Beaulac K, Corcione S, Epstein L, Davidson LE, Doron S. Antimicrobial stewardship in a long-term acute care hospital using offsite electronic medical record audit. Infect Control Hosp Epidemiol. 2016;37:433-9.

15. dos Santos RP, Deutschendorf C, Carvalho OF, Timm R, Sparenberg A. Antimicrobial stewardship through telemedicine in a community hospital in southern Brazil. J Telemed Telecare. 2013;19:1-4.

16. Wilson BM, Banks RE, Crnich CJ, Ide E, Viau RA, El Chakhtoura NG, et al. Changes in antibiotic use following implementation of a telehealth stewardship pilot program. Infect Control Hosp Epidemiol. 2019;40:810-4.

17. Yam P, Fales D, Jemison J, Gillum M, Bernstein M. Implementation of an antimicrobial stewardship program in a rural hospital. Am J Heal Pharm. 2012;69:1142-8.

18. Ceradini J, Tozzi AE, D'Argenio P, Bernaschi P, Manuri L, Brusco C, et al. Telemedicine as an effective intervention to improve antibiotic appropriateness prescription and to reduce costs in pediatrics. Ital J Pediatr. Italian Journal of Pediatrics; 2017;43:10-3.

19. Du Yan L, Dean K, Park D, Thompson J, Tong I, Liu $\mathrm{C}$, et al. Education vs clinician feedback on antibiotic prescriptions for acute respiratory infections in telemedicine: a randomized controlled trial. J Gen Intern Med. Journal of General Internal Medicine; 2020;

20. Wood ZH, Nicolsen NC, Allen N, Cook PP. Remote antimicrobial stewardship in community hospitals. Antibiotics. 2015;4:605-16.

21. Morquin D, Ologeanu-Taddei R, Koumar Y, Reynes J. Tele-expertise system based on the use of the electronic patient record to support real-time antimicrobial use. Int J Technol Assess Health Care. 2018;34:156-62.

22. Howell CK, Jacob J, Mok S. Remote antimicrobial stewardship: a solution for meeting the joint commission stewardship standard? Hosp Pharm. 2019;54:51-6.

23. Shively NR, Moffa MA, Paul KT, Wodusky EJ, Schipani BA, Cuccaro SL, et al. Impact of a telehealth-based antimicrobial stewardship program in a community hospital health system. Clin Infect Dis. 2019;1-7. doi:https://doi.org/10.1093/cid/ciz878

24. Echevarria K, Groppi J, Kelly AA, Morreale AP, Neuhauser MM, Roselle GA. Development and application of an objective staffing calculator for antimicrobial stewardship programs in the Veterans Health Administration. Am J Heal Pharm. 2017;74:1785-90.

25. Doernberg SB, Abbo LM, Burdette SD, Fishman NO, Goodman EL, Kravitz GR, et al. Essential resources and strategies for antibiotic stewardship programs in the acute care setting. Clin Infect Dis. 2018;67:1168-74.

26. NRMP. NRMP Results and data specialties matching service, 2020 appointment year [Internet]. 2020. Available from: https://mk0nrmp3oyqui6wqfm. kinstacdn.com/wp-content/uploads/2020/02/Resul ts-and-Data-SMS-2020.pdf

27. CMS. Trump administration drives telehealth services in medicaid and medicare [Internet]. 2020 [cited 2020 Nov 9]. Available from: https://www. cms.gov/newsroom/press-releases/trump-administra tion-drives-telehealth-services-medicaid-and-medic are

\section{Publisher's Note}

Springer Nature remains neutral with regard to jurisdictional claims in published maps and institutional affiliations. 